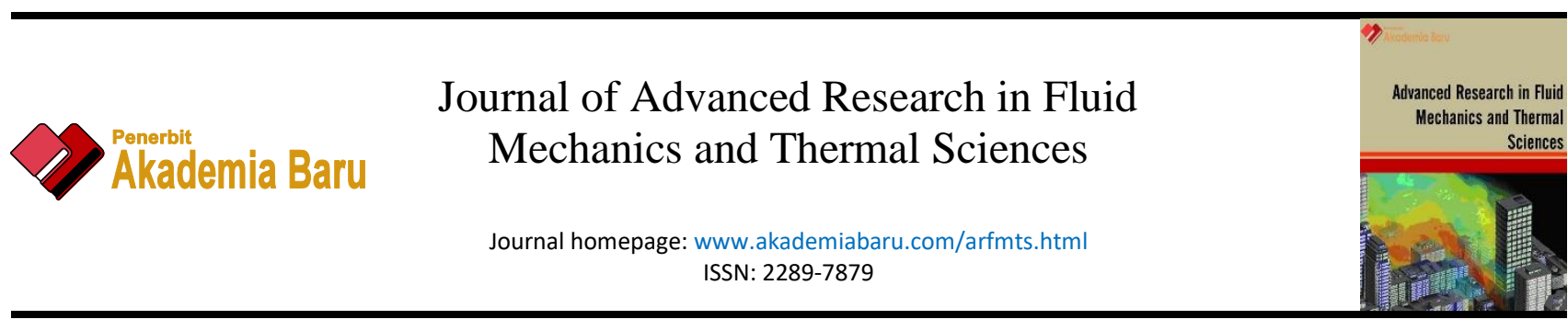

\title{
Relaxation Equations of Consolidating Cake Filtration
}

\author{
Bakhtiyor Khuzhayorov ${ }^{1, *}$, Usmonali Saydullaev ${ }^{1}$, Bekzodjon Fayziev ${ }^{1}$ \\ Department of Mathematical Modeling, Samarkand State University, 15, University Str., Samarkand City, 140100, Republic of Uzbekistan
}

ARTICLE INFO
ABSTRACT

\section{Article history:}

Received 29 March 2020

Received in revised form 25 May 2020

Accepted 4 June 2020

Available online 15 August 2020

\section{Keywords:}

Cake filtration; filter; relaxation; relaxing cake; Stefan problem; suspension

\begin{abstract}
In this paper, on the basis of conservation laws suspensions filtration equations with forming a relaxing cake are derived. The equations are numerically solved. To solve the equation for cake growth a Stefan problem is posed and solved with using the method of catching a moving front. On the basis of numerical results influence of relaxation phenomena on filtration characteristics is established. It is shown that the relaxation slows down the increasing of local filter cake porosity and decreasing of cake permeability, as well as increase fluid relative velocity through cake for given applied pressure. Increasing of relaxation time leads to a faster growth of the cake thickness when all other conditions are constants, thus relaxation effects cause more intensive transfer of particles from suspension to the cake. It, in turn, alters all other filtration characteristics, such as fluid pressure distribution, compressive pressure, porosity and consolidation of the cake, fluid flow rate through the cake and effective hydrodynamic resistance. With increasing of relaxation phenomena dynamics of current and total outlet filtrate flow rate becomes more intensive.
\end{abstract}

Copyright $@ 2020$ PENERBIT AKADEMIA BARU - All rights reserved

\section{Introduction}

Filtration refers to the processes of separation of heterogeneous systems using a porous medium (filter), which hold up one phase of these systems and let others pass through. These processes include separation of the suspension into pure liquid and solid particles. Processes of suspensions separation have wide applications in chemical, petrochemical, oil and gas, coal, food and some other industries. At filtration, solid particles contained in the suspension are retained on the surface of the filter and form a cake. This process is called as filtering with formation of a cake [1-7]. The cake formed during the filtration of different suspensions usually is a complex multi-component system consisting, in general, of liquid and solid particles of various shapes and sizes. Solid particles of the cake are under the influence of external and internal forces and interact with the surrounding medium and with each other. External fields influencing on cake particles include pressure drop and

\footnotetext{
${ }^{*}$ Corresponding author.

E-mail address: b.khuzhayorov@mail.ru
}

https://doi.org/10.37934/arfmts.74.2.168182 
gravitational forces. Internal fields are excited by inter particle interaction forces. These include: the forces of chemical nature, molecular, ion-electrostatic, capillary and magnetic forces [8-10]. To study the influence of these factors is very important in the design and construction of different filtering elements, for instance, ultrafiltration membranes (UF). UF has been recognized as one of the advanced technologies and is widely implemented in various field of industry. The performance of sandwich membrane with different configurations is studied in [11]. Separation properties and performance in membrane reactor systems are studied in [12].

The filtration with cake formation is based on the fundamental equations of the mechanics of multi-component media, the theory of filtration consolidation [13-20] and takes into account the influence of hydrodynamic and rheological factors. Differential equations of filtering are obtained on the basis of the two-phase flows mechanics equations, taking into account the changes in the filter parameters along the thickness of the cake with the time $[9,10,21,22]$. Since the cake-suspension interface is mobile, the equation describing the compressibility of the cake leads to Stefan's problems $[14,23,24]$.

When we consider non-Newtonian systems, such as, highly concentrated suspensions, as well as suspensions with high-molecular polymers, the classical Darcy's law is violated. In this case, the Darcy law is nonlinear or non-equilibrium. At filtration of dispersed systems in porous media due to deposition of some phase components in the pore space, the filtration flow can have relaxation behaviour. A number of works are devoted to this phenomenon [25-31]. In this case, we have a nonequilibrium relationship between the filtration velocity and the pressure gradient. Relaxation phenomena in the filtration of liquids are reflected in the equations used for mathematical modeling of the filtration process. Usually, in the description of relaxation filtration, various phenomenological models are used, taking into account the delay in the dependences between the filtration velocity and the pressure gradient. In some cases, it is necessary also to take into account the delay in the equations of state. In our case liquid phase of suspension, we treat as polymer solution that exhibits non-equilibrium rheological properties, as a consequence, it leads to a non-equilibrium filtration law. So, we use here relaxation type filtration law, where pressure gradient relaxes with respect the filtration velocity.

As one of the first in this direction, one can mention the work [32]. In this work on the basis of numerous experimental data, a relaxation changes in the velocity of filtration during the flow of a polymer solution through a porous medium at a constant pressure drop is shown. Khuzhayorov [31] research on some non-steady one-dimensional filtration problems under the elastic regime are considered with an assumption that the pressure gradient has relaxation behaviour with respect to the velocity of filtration. A systematic analysis of the relaxation theory of filtration is given in the research by Molokovich [34].

As far as we know, still the relaxation phenomena in the theory of cake filtration are not applied. However, it is important both in theoretical and practical point of view. The studies of relaxation phenomena allow us better understanding of suspensions separation processes when liquid phase of suspension has high viscosity and anomalous rheological properties.

In this paper, we consider only phenomenological models for the filtration of disperse systems. Macroscopic modeling has recently been very intensively developed, which yet is not practically used yet in filtration problems of disperse systems. Some macroscopic models of relaxation mass transport in a porous medium are derived and analyzed in the research by Khuzhayorov [35]. We first derive cake filtration equations and an equation for the cake growth. Then we perform numerical analyses of equations and present results. On the basis of the last we make some conclusions. Note, here we emphasize our attention only on the relaxation effects in the filtration law. In general, relaxation effects can have other nature, for instance cake properties can have non-equilibrium dependences. 


\section{Mathematical Model}

\subsection{Derivation of Cake Filtration Equations}

We consider cake filtration of suspensions (Figure 1). At filtration of suspensions with forming a cake, the Darcy's law establishes an equilibrium relationship between the relative velocity $q_{l s}$ and the pressure gradient $\partial p_{l} / \partial x$, which in one-dimensional scalar form can be written as [5]

$\frac{q_{l s}}{\varepsilon}=\frac{q_{l}}{\varepsilon}-\frac{q_{s}}{\varepsilon}=-\frac{1}{\varepsilon} \frac{k}{\mu} \frac{\partial p_{l}}{\partial x}$

where $l$ is index corresponding to the liquid phase, $s$ is index corresponding to solid particles, $\varepsilon_{i}$ is porosity of the $i$-th phase (here and after we use $\varepsilon$ instead of $\varepsilon_{l}$ for simplicity), $q_{l}$ is liquid phase velocity, $q_{s}$ is solid phase velocity, $p_{l}$ is pressure in the liquid phase, $k$ is permeability coefficient, $\mu$ is viscosity.

Suppose that this relationship is of a non-equilibrium nature. Non-equilibrium nature can occur both in the filtration velocity of the liquid and solid phases relative to the pressure gradient, and vice versa, in the pressure gradient relative to the filtration velocities. The non-equilibrium relationship is assumed in the linear differential form. In addition, since the velocities of solid and liquid phases can have different scales of variation, the relaxation effects can also occur with different characteristic times.

Following to this idea the relaxation filtering law we take in the form

$$
\left(1+\lambda_{q l} \frac{\partial}{\partial t}\right) \frac{q_{l}}{\varepsilon}-\left(1+\lambda_{q s} \frac{\partial}{\partial t}\right) \frac{q_{s}}{\varepsilon_{s}}=-\frac{1}{\varepsilon} \frac{k}{\mu}\left(1+\lambda_{p l} \frac{\partial}{\partial t}\right) \frac{\partial p_{l}}{\partial x}
$$

where $\lambda_{q l}$ is the relaxation time of filtration velocities, $\lambda_{p l}$ is the relaxation time of the pressure gradient.

In the cake, the velocity of the particles is much lower than the velocity of the liquid phase. The velocity of particles at the boundary of the suspension and the cake can be assumed to be equal to the velocity of the suspension, and at the boundary of the cake and the filter surface, this velocity is zero. So, the filtration velocity of solid particles decreases along the cake from the velocity of the suspension to zero. The velocity of the liquid phase increases along the cake from the boundary with the suspension to the boundary of the filter, while the overall filtration velocity $q_{l}+q_{s}$ remains constant with respect to $x$. Because of this, the change rate in the velocity of filtration of the solid phase compared with the liquid phase can be considered to be slow. This circumstance makes it possible to neglect the relaxation effects in the filtration velocity of the solid phase in comparison with the liquid phase. Then Eq. (1) can be written in the form

$$
\left(1+\lambda_{q l} \frac{\partial}{\partial t}\right) \frac{q_{l}}{\varepsilon}-\frac{q_{s}}{\varepsilon_{s}}=-\frac{1}{\varepsilon} \frac{k}{\mu}\left(1+\lambda_{p l} \frac{\partial}{\partial t}\right) \frac{\partial p_{l}}{\partial x},
$$

For the sake of simplicity, let us first consider only relaxation of the pressure gradient, i.e. we take $\lambda_{q l}=0$. Then Eq. (1) takes the form

$$
\frac{q_{l}}{\varepsilon}-\frac{q_{s}}{\varepsilon_{s}}=-\frac{1}{\varepsilon} \frac{k}{\mu}\left(1+\lambda_{p l} \frac{\partial}{\partial t}\right) \frac{\partial p_{l}}{\partial x} .
$$

At $x=0$ by taking into account $\left.q_{s}\right|_{x=0}=0$ from Eq. (3) we have 
$\left.q_{l}\right|_{x=0}=q_{l m}=-\left.\frac{k}{\mu}\left(1+\lambda_{p l} \frac{\partial}{\partial t}\right) \frac{\partial p_{l}}{\partial x}\right|_{x=0^{\prime}}$

where $q_{l m}=0$ is the instantaneous filtration velocity at $x=0$. Because $\left.q_{s}\right|_{x=0}=0$, the term $q_{l m}$ represents, in fact, filtrate velocity.

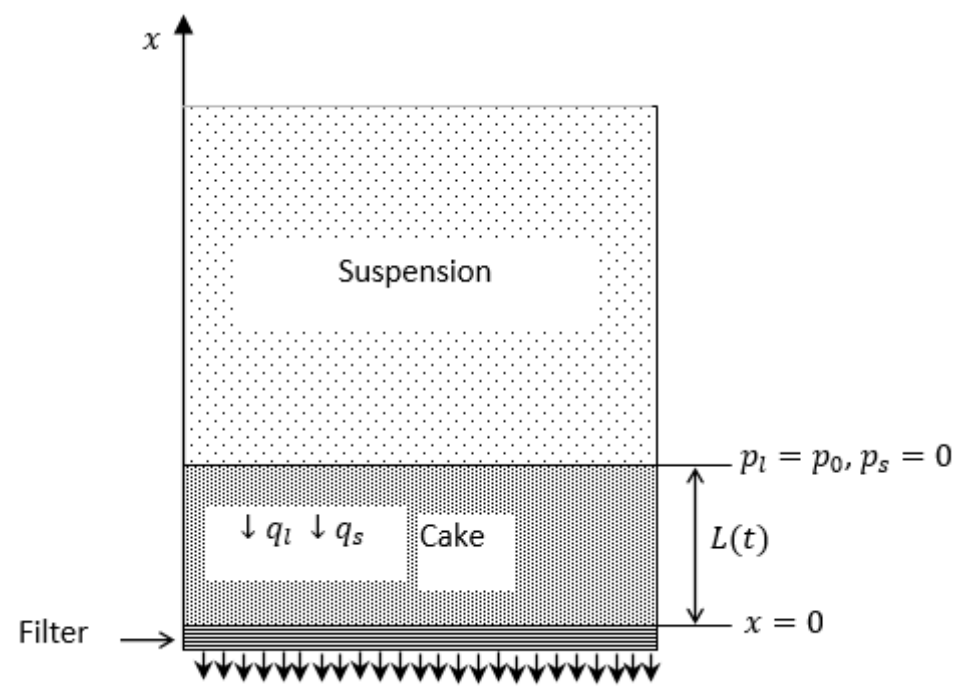

Fig. 1. A schematic of cake filtration

The flow $q_{l m}$ is balanced by the flow through the filter, so in Eq. (4) we have

$\left.q_{l}\right|_{x=0}=-\left.\frac{k}{\mu}\left(1+\lambda_{p l} \frac{\partial}{\partial t}\right) \frac{\partial p_{l}}{\partial x}\right|_{x=0}=-\frac{p_{l m}}{\mu R_{m}}$,

where $p_{l m}$ is the filtrate pressure at $x=0, R_{m}$ is relative resistance of the filtering element. We transform Eq. (3) into

$\frac{q_{l}}{\varepsilon}-\frac{q_{l m}-q_{l}}{\varepsilon_{S}}=-\frac{1}{\varepsilon} \frac{k}{\mu}\left(1+\lambda_{p l} \frac{\partial}{\partial t}\right) \frac{\partial p_{l}}{\partial x}$

and from that we have

$q_{l}=\varepsilon q_{l m}-\varepsilon_{s} \frac{k}{\mu}\left(1+\lambda_{p l} \frac{\partial}{\partial t}\right) \frac{\partial p_{l}}{\partial x}$.

By taking into account Eq. (4), from Eq. (6) we obtain

$q_{l}=\left(1-\varepsilon_{S}\right)\left[\frac{k}{\mu}\left(1+\lambda_{p l} \frac{\partial}{\partial t}\right) \frac{\partial p_{l}}{\partial x}\right]_{x=0}-\varepsilon_{S}\left[\frac{k}{\mu}\left(1+\lambda_{p l} \frac{\partial}{\partial t}\right) \frac{\partial p_{l}}{\partial x}\right]$.

To derive a filtration equation, we use the following balance equation

$\frac{\partial \varepsilon}{\partial t}=-\frac{\partial q_{l}}{\partial x}$

After substitution Eq. (7) into Eq. (8) and with taking into account $\varepsilon=1-\varepsilon_{s}$ we have 
$-\frac{\partial \varepsilon_{s}}{\partial t}=-\frac{\partial}{\partial x}\left\{-\varepsilon_{S} \frac{k}{\mu}\left(1+\lambda_{p l} \frac{\partial}{\partial t}\right) \frac{\partial p_{l}}{\partial x}-\left(1-\varepsilon_{s}\right)\left[\frac{k}{\mu}\left(1+\lambda_{p l} \frac{\partial}{\partial t}\right) \frac{\partial p_{l}}{\partial x}\right]_{x=0}\right\}$

or

$-\frac{\partial \varepsilon_{s}}{\partial t}=\frac{\partial}{\partial x}\left\{-\varepsilon_{s} \frac{k}{\mu}\left(1+\lambda_{p l} \frac{\partial}{\partial t}\right) \frac{\partial p_{l}}{\partial p_{s}} \frac{\partial p_{s}}{\partial x}\right\}-q_{l m} \frac{\partial \varepsilon_{s}}{\partial x}$,

where

$q_{l m}=-\left.\frac{k}{\mu}\left(1+\lambda_{p l} \frac{\partial}{\partial t}\right) \frac{\partial p_{l}}{\partial x}\right|_{x=0}$.

There are different relations between $p_{l}$ and $p_{s}$. Some of the simplest possible relations are [5]:

1) Type 1: $d p_{l}+d p_{s}=0$;

2) Type 2: $\left(1-\varepsilon_{s}\right) d p_{l}+d p_{s}=0$;

3) Type 3: $\left(1-\varepsilon_{s}\right) d p_{l}+\varepsilon_{s} d p_{s}=0$;

4) Type 4: $d\left[\left(1-\varepsilon_{s}\right) d p_{l}\right]+d\left[\varepsilon_{s} d p_{s}\right]=0$.

The general representation of these results can be written as [5]; $\frac{\partial p_{l}}{\partial p_{s}}=f^{\prime}$, where $f^{\prime}$ for given above types is:

1) Type 1: $f^{\prime}=-1$;

2) Type 2: $f^{\prime}=-\frac{1}{1-\varepsilon_{s}}$;

3) Type 3: $f^{\prime}=-\frac{\varepsilon_{s}}{1-\varepsilon_{s}}$;

4) Type 4: $f^{\prime}=-\frac{\left(1-\varepsilon_{S}\right) p_{0}-p_{s}}{\left(1-\varepsilon_{S}\right)^{2}} \frac{d \varepsilon_{s}}{d p_{s}}-\frac{\varepsilon_{s}}{1-\varepsilon_{S}}$.

So, from Eq. (9) we have

$-\frac{\partial \varepsilon_{s}}{\partial t}=\frac{\partial}{\partial x}\left\{-\varepsilon_{s} \frac{k}{\mu}\left(1+\lambda_{p l} \frac{\partial}{\partial t}\right) f^{\prime} \frac{\partial p_{s}}{\partial x}\right\}-q_{l m} \frac{\partial \varepsilon_{s}}{\partial x}$.

If we use the relations [5] $\varepsilon_{s}=\varepsilon_{s}^{0}\left(1+\frac{p_{s}}{p_{l}}\right)^{\beta}$ and $k=k^{0}\left(1+\frac{p_{s}}{p_{l}}\right)^{-\delta}$ from Eq. (10) we obtain the following equation with respect to $p_{S}$

$\frac{\varepsilon_{s}^{0} \beta}{p_{A}}\left(1+\frac{p_{s}}{p_{l}}\right)^{\beta-1} \frac{\partial p_{s}}{\partial t}=-\frac{k^{0} \varepsilon_{s}^{0}}{\mu} \frac{\partial}{\partial x}\left[\left(1+\frac{p_{s}}{p_{l}}\right)^{\beta-\delta}\left(1+\lambda_{p l} \frac{\partial}{\partial t}\right)\left(f^{\prime} \frac{\partial p_{s}}{\partial x}\right)\right]-\frac{\varepsilon_{s}^{0} \beta}{p_{A}} q_{l m}\left(1+\frac{p_{s}}{p_{l}}\right)^{\beta-1} \frac{\partial p_{s}}{\partial t}$,

where $\varepsilon_{S}^{0}$ and $k^{0}$ denote respectively the values of value of $\varepsilon_{S}$ and $k$ at $p_{S}=0, p_{A}=0$ - characteristic pressure, $\beta, \delta$ signifies the compression effect due to $p_{s}$.

In the case $f^{\prime}=-1$ (type 1 ) from Eq. (11) we have

$\frac{\varepsilon_{s}^{0} \beta}{p_{A}}\left(1+\frac{p_{s}}{p_{l}}\right)^{\beta-1} \frac{\partial p_{s}}{\partial t}=\frac{k^{0} \varepsilon_{s}^{0}}{\mu} \frac{\partial}{\partial x}\left[\left(1+\frac{p_{s}}{p_{l}}\right)^{\beta-\delta}\left(1+\lambda_{p l} \frac{\partial}{\partial t}\right) \frac{\partial p_{s}}{\partial x}\right]-\frac{\varepsilon_{s}^{0} \beta}{p_{A}} q_{l m}\left(1+\frac{p_{s}}{p_{l}}\right)^{\beta-1} \frac{\partial p_{s}}{\partial t}$,

where expression for $q_{l m}$ with respect to $p_{s}$ is

$q_{l m}=\left.\frac{k}{\mu}\left(1+\lambda_{p l} \frac{\partial}{\partial t}\right) \frac{\partial p_{s}}{\partial x}\right|_{x=0}$. 
Eq. (12) is the basic equation of non-equilibrium filtration with cake formation. It can be solved with corresponding initial and boundary conditions, specified in particular on the moving boundary $x=L(t)$, which must be determined from the additional equation.

The initial and boundary conditions for Eq. (12) can be taken in the form

$p_{s}(0, x)=0, p_{s}(t, L(t))=0,-\left.k\left(1+\lambda_{p l} \frac{\partial}{\partial t}\right) \frac{\partial p_{s}}{\partial x}\right|_{x=0}=\left.\frac{p_{0}-p_{s}}{R_{m}}\right|_{x=0}$,

where $p_{0}$ - suspension feed pressure.

\subsection{Derivation of Equation for The Cake Growth}

Now we proceed to the derivation of an equation for the mobile boundary $L(t)$, which characterizes the growth of the cake. The mobile boundary $L(t)$ expresses the thickness of the cake and continuously grows during the filtration process. The mobile boundary is determined on the basis of the mass conservation law on the surface of the cake, i.e. on the common boundary of the suspension and the cake. At $x<L(t)$ we have the cake, and at $x=L(t)$ we have the suspension. Then, the basic equation characterizing the growth of the cake can be taken in the form $[5,6]$

$\frac{d L}{d t}=\frac{-\left.q_{l}\right|_{L^{+}}-\left(-\left.q_{l}\right|_{L^{-}}\right)}{\varepsilon_{S_{0}}-\varepsilon_{S}^{0}}$

where $\varepsilon_{S}^{0}$ is solid content at zero pressure, $\varepsilon_{S_{0}}$ is concentration of solid particles in suspension, $\left.q_{l}\right|_{L^{+}}$ is filtration velocity at $x=L^{+},\left.q_{l}\right|_{L^{-}}$is filtration velocity at $x=L^{-}$.

For the relaxation filtration law, we have the following relationships

$\left.q_{l}\right|_{L^{+}}+\left.q_{s}\right|_{L^{+}}=\left.q_{l}\right|_{L^{-}}+\left.q_{s}\right|_{L^{-}}=q_{l m}=\left[-\frac{k}{\mu}\left(1+\lambda_{p l} \frac{\partial}{\partial t}\right) \frac{\partial p_{l}}{\partial x}\right]_{x=0}$.

The Darcy's law at $x=L^{-}$can be written as

$\left.q_{l}\right|_{L^{-}}-\left.\frac{1-\varepsilon_{s}^{0}}{\varepsilon_{s}^{0}} q_{s}\right|_{L^{-}}=\left[-\frac{k}{\mu}\left(1+\lambda_{p l} \frac{\partial}{\partial t}\right) \frac{\partial p_{l}}{\partial x}\right]_{x=L^{-}}$.

From Eq. (15) we obtain

$\left.q_{l}\right|_{L^{-}}=-\left.q_{S}\right|_{L^{-}}-\left[\frac{k}{\mu}\left(1+\lambda_{p l} \frac{\partial}{\partial t}\right) \frac{\partial p_{l}}{\partial x}\right]_{x=0}$.

Eq. (16) and Eq. (17) give

$\left.q_{l}\right|_{L^{-}}=-\varepsilon_{S}^{0}\left[\frac{k}{\mu}\left(1+\lambda_{p l} \frac{\partial}{\partial t}\right) \frac{\partial p_{l}}{\partial x}\right]_{x=L^{-}}-\left(1-\varepsilon_{s}^{0}\right)\left[\frac{k}{\mu}\left(1+\lambda_{p l} \frac{\partial}{\partial t}\right) \frac{\partial p_{l}}{\partial x}\right]_{x=0}$.

In the suspension zone $x=L^{+}$particles and liquid move with equal velocities

$\frac{\left.q_{s}\right|_{L^{+}}}{\varepsilon_{S_{0}}}=\frac{\left.q_{l}\right|_{L^{+}}}{1-\varepsilon_{S_{0}}}$ 
that gives

$\left.q_{s}\right|_{L^{+}}=\left.\frac{\varepsilon_{S_{0}}}{1-\varepsilon_{S_{0}}} q_{l}\right|_{L^{+}}$

By substitution Eq. (19) into Eq. (15) we obtain

$\left.q_{l}\right|_{L^{+}}=-\left(1-\varepsilon_{S_{0}}\right)\left[\frac{k}{\mu}\left(1+\lambda_{p l} \frac{\partial}{\partial t}\right) \frac{\partial p_{l}}{\partial x}\right]_{x=0}$.

With taking into account Eq. (18) and Eq. (20), from the Eq. (14) we have

$\frac{d L}{d t}=\frac{\varepsilon_{S}^{0}}{\varepsilon_{s}^{0}-\varepsilon_{S_{0}}}\left[\frac{k}{\mu}\left(1+\lambda_{p l} \frac{\partial}{\partial t}\right) \frac{\partial p_{l}}{\partial x}\right]_{L^{-}}+q_{l m}$.

If we use the first type of the relationship between $p_{l}$ and $p_{s}$, i.e. $f^{\prime}=-1$, the Eq. (21) can be written with respect to $p_{s}$

$\frac{d L}{d t}=-\frac{\varepsilon_{S}^{0}}{\varepsilon_{S}^{0}-\varepsilon_{S_{0}}}\left[\frac{k}{\mu}\left(1+\lambda_{p l} \frac{\partial}{\partial t}\right) \frac{\partial p_{s}}{\partial x}\right]_{L^{-}}+q_{l m}$.

By integrating the Eq. (22) the mobile front $L(t)$ can be determined. This equation is solved together with the basic equation of filtration Eq. (12) with Eq. (13).

We should note, that without relaxation effects, the analogous problems of filtration were considered in $[5,9,10]$.

\section{Numerical Analysis}

Eq. (12), we write in the following form

$$
\frac{\partial p_{s}}{\partial t}=\frac{k^{0} p_{A}}{\beta \mu}\left(1+\frac{p_{s}}{p_{l}}\right)^{1-\beta} \frac{\partial}{\partial x}\left[\left(1+\frac{p_{s}}{p_{l}}\right)^{\beta-\delta}\left(1+\lambda_{p l} \frac{\partial}{\partial t}\right) \frac{\partial p_{s}}{\partial x}\right]-q_{l m} \frac{\partial p_{s}}{\partial t}
$$

We introduce the following notations

$$
\begin{aligned}
& a(p)=\left(1+\frac{p_{s}}{p_{l}}\right)^{1-\beta}, b(p)=\left(1+\frac{p_{s}}{p_{l}}\right)^{\beta-\delta}, c_{2}=\frac{\varepsilon_{s}^{0}}{\varepsilon_{s}^{0}-\varepsilon_{s_{0}}}, c(p)=\frac{k^{0}}{\mu}\left(1+\frac{p_{s}}{p_{l}}\right)^{-\delta}, c_{1}=\frac{k^{0} p_{A}}{\beta \mu}, c^{0}(p)= \\
& \left.\frac{k^{0}}{\mu}\left(1+\frac{p_{s}}{p_{l}}\right)^{-\delta}\right|_{x=0} .
\end{aligned}
$$

With taking into account these notations, Eq. (23) can be transformed into the following form

$$
\frac{\partial p_{s}}{\partial t}=c_{1} a(p) \frac{\partial}{\partial x}\left[b(p)\left(1+\lambda_{p l} \frac{\partial}{\partial t}\right) \frac{\partial p_{s}}{\partial x}\right]-q_{l m} \frac{\partial p_{s}}{\partial t} .
$$

The equation for the mobile boundary $L(t)$, Eq. (22), takes the form 
$\frac{d L}{d t}=-c_{2}\left[c(p)\left(1+\lambda_{p l} \frac{\partial}{\partial t}\right) \frac{\partial p_{s}}{\partial x}\right]_{L^{-}}+q_{l m}$

where

$q_{l m}=\left.c^{0}(p)\left(1+\lambda_{p l} \frac{\partial}{\partial t}\right) \frac{\partial p_{s}}{\partial x}\right|_{x=0}$.

To solve the problem Eq. (24), Eq. (25) with Eq. (13) and $L(0)=0$, we use the finite differences method. We introduce a uniform grid by $t$ with the step $\tau \bar{\omega}_{\tau}=\left\{t \mid t=t_{j}=j \tau, j=0,1, \ldots, N, \tau N=\right.$ $T\}$, and a non - uniform grid by coordinate $x[36,37]$

$\bar{\omega}_{h}=\left\{x x=x_{i}=x_{i-1}+h_{i}, i=0,1, \ldots, N, N+1, \ldots x_{N}=L\right\}$

with the variable step $h_{i}>0$.

We are to choose the step $h_{i}$ from the section $\left[x_{i}, x_{i+1}\right]$ so, that the mobile boundary moves exactly on one step along the time grid. This approach is known as the method of catching the front in a grid node. We denote by $\phi_{i}^{j+1}$ the grid function corresponding to $p_{s}$. We approximate Eq. (24) by an implicit difference scheme that is nonlinear with respect to the function $\phi_{i}^{j+1}$

$$
\begin{aligned}
& \frac{\phi_{i}^{j+1}-\phi_{i}^{j}}{\tau}=c_{1} \frac{2 a\left(\phi_{i}^{j}\right)}{h_{i}+h_{i+1}} \frac{\partial}{\partial x}\left\{b\left(\phi_{i+\frac{1}{2}}^{j+1}\right) \frac{\phi_{i+1}^{j+1}+\phi_{i-1}^{j+1}}{h_{i}+h_{i+1}}+\frac{\lambda_{p l}}{\tau} b\left(\phi_{i+\frac{1}{2}}^{j+1}\right)\left[\frac{\phi_{i+1}^{j+1}+\phi_{i-1}^{j+1}}{h_{i}+h_{i+1}}-\right.\right. \\
& \left.\left.\frac{\phi_{i+1}^{j}+\phi_{i-1}^{j}}{h_{i}+h_{i+1}}\right]-b\left(\phi_{i-\frac{1}{2}}^{j+1}\right) \frac{\phi_{i}^{j+1}+\phi_{i}^{j+1}}{h_{i}}--\frac{\lambda_{p l}}{\tau} b\left(\phi_{i-\frac{1}{2}}^{j+1}\right)\left[\frac{\phi_{i}^{j+1}+\phi_{i-1}^{j+1}}{h_{i}}-\frac{\phi_{i}^{j}+\phi_{i-1}^{j}}{h_{i}}\right]\right\}-\left(q_{l m}\right)_{0}^{j+1} \frac{\phi_{i}^{j+1}+\phi_{i-1}^{j+1}}{h_{i}}, \\
& i=1, \ldots N-1, j=0,1, \ldots N-1,
\end{aligned}
$$

where

$$
\begin{aligned}
& a\left(\phi_{i}^{j}\right)=\left(1+\frac{\phi_{i}^{j}}{p_{A}}\right)^{1-\beta}, b\left(\phi_{i+\frac{1}{2}}^{j}\right)=\frac{1}{2}\left[\left(1+\frac{\phi_{i+1}^{j+1}}{p_{A}}\right)^{\beta-\delta}+\left(1+\frac{\phi_{i}^{j+1}}{p_{A}}\right)^{\beta-\delta}\right], \\
& \left(q_{l m}\right)_{0}^{j+1}=c^{0}\left(\phi_{0}^{j+1}\right)\left(\frac{\phi_{1}^{j+1}-\phi_{0}^{j+1}}{h_{0}}+\frac{\lambda_{p l}}{\tau}\left(\frac{\phi_{1}^{j+1}-\phi_{0}^{j+1}}{h_{0}}-\frac{\phi_{1}^{j}-\phi_{0}^{j}}{h_{0}}\right)\right), c^{0}\left(\phi_{0}^{j+1}\right)=\frac{k^{0}}{\mu}\left(1+\frac{\phi_{0}^{j+1}}{p_{A}}\right)^{-\delta} .
\end{aligned}
$$

Eq. (25) when $\frac{d L}{d t} \approx \frac{h_{i+1}}{\tau}$ after the approximation can be written in the form

$$
\frac{h_{i+1}}{\tau}=-c_{2}\left[c^{0}\left(\phi_{i-\frac{1}{2}}^{j+1}\right)\left(\frac{\phi_{i}^{j+1}-\phi_{i-1}^{j+1}}{h_{i+1}}+\frac{\lambda_{p l}}{\tau}\left(\frac{\phi_{i}^{j+1}-\phi_{i-1}^{j+1}}{h_{i+1}}-\frac{\phi_{i}^{j}-\phi_{i-1}^{j}}{h_{i+1}}\right)\right)\right]+\left(q_{l m}\right)_{0}^{j+1},
$$

where

$$
c\left(\phi_{i-\frac{1}{2}}^{j}\right)=\frac{k^{0}}{2 \mu}\left[\left(1+\frac{\phi_{i}^{j}}{p_{A}}\right)^{-\delta}+\left(1+\frac{\phi_{i-1}^{j}}{p_{A}}\right)^{-\delta}\right] .
$$

Approximation of initial and boundary conditions Eq. (13) gives 


$$
\begin{array}{r}
-\mu c^{0}\left(\phi_{0}^{j}\right)\left(\frac{\phi_{1}^{j+1}-\phi_{0}^{j+1}}{h_{1}}+\frac{1}{\tau}\left(\frac{\phi_{1}^{j+1}-\phi_{0}^{j+1}}{h_{1}}-\frac{\phi_{1}^{j}-\phi_{0}^{j}}{h_{1}}\right)\right)=\frac{p_{0}+\phi_{0}^{j+1}}{R_{m}}, j=\overline{0, N} \\
\phi_{i}^{j+1}=0, i=N+1, N+2, \ldots, j=0,1, \ldots
\end{array}
$$

The obtained set of Eq. (26) is nonlinear, so to solve it we use the method of simple iteration

$$
\begin{aligned}
& \frac{\left(\phi^{(s+1)}\right)_{i}^{j+1}-\phi_{i}^{j}}{\tau}=c_{1} \frac{2 a\left(\phi_{i}^{j}\right)}{h_{i}+h_{i+1}} \frac{\partial}{\partial x}\left\{b\left(\left(\phi^{(s)}\right)_{i+\frac{1}{2}}^{j+1}\right) \frac{\left(\phi^{(s+1)}\right)_{i+1}^{j+1}+\left(\phi^{(s+1)}\right)_{i-1}^{j+1}}{h_{i}+h_{i+1}}+\right. \\
& \frac{\lambda_{p l}}{\tau} b\left(\left(\phi^{(s)}\right)_{i+\frac{1}{2}}^{j+1}\right)\left[\frac{\left(\phi^{(s+1)}\right)_{i+1}^{j+1}+\left(\phi^{(s+1)}\right)_{i-1}^{j+1}}{h_{i}+h_{i+1}}-\frac{\phi_{i+1}^{j}+\phi_{i-1}^{j}}{h_{i}+h_{i+1}}\right]-b\left(\left(\phi^{(s)}\right)_{i-\frac{1}{2}}^{j+1} \frac{\left(\phi^{(s+1)}\right)_{i}^{j+1}+\left(\phi^{(s+1)}\right)_{i}^{j+1}}{h_{i}}-\right. \\
& \left.\frac{\lambda_{p l}}{\tau} b\left(\left(\phi^{(s)}\right)_{i-\frac{1}{2}}^{j+1}\right)\left[\frac{\left(\phi^{(s+1)}\right)_{i}^{j+1}+\left(\phi^{(s+1)}\right)_{i-1}^{j+1}}{h_{i}}-\frac{\phi_{i}^{j}+\phi_{i-1}^{j}}{h_{i}}\right]\right\}-\left(q_{l m}^{(s)}\right)_{0}^{j+1} \frac{\left(\phi^{(s+1)}\right)_{i}^{j+1}+\left(\phi^{(s+1)}\right)_{i-1}^{j+1}}{h_{i}},
\end{aligned}
$$

where

$$
\begin{aligned}
& b\left(\left(\phi^{(s)}\right)_{i+\frac{1}{2}}^{j}\right)=\frac{1}{2}\left[\left(1+\frac{\left(\phi^{(s)}\right)_{i+1}^{j+1}}{p_{A}}\right)^{\beta-\delta}+\left(1+\frac{\left(\phi^{(s)}\right)_{i}^{j+1}}{p_{A}}\right)^{\beta-\delta}\right], \\
& \left(q_{l m}^{(s)}\right)_{0}^{j+1}=c^{0}\left(\left(\phi^{(s)}\right)_{0}^{j+1}\right)\left(\frac{\left(\phi^{(s)}\right)_{1}^{j+1}-\left(\phi^{(s)}\right)_{0}^{j+1}}{h_{0}}+\frac{\lambda_{p l}}{\tau}\left(\frac{\left(\phi^{(s)}\right)_{1}^{j+1}-\left(\phi^{(s)}\right)_{0}^{j+1}}{h_{0}}-\frac{\phi_{1}^{j}-\phi_{0}^{j}}{h_{0}}\right)\right),
\end{aligned}
$$

$s$ is the number of iteration.

It can be seen that the system of Eq. (29) is now linear with respect to $\left(\phi^{(s+1)}\right)_{i}^{j+1}$, which allows us to use the Tomas' algorithm. As a condition to stop iteration procedure on this time layer, the following relationship can be used:

$\max _{i}\left|\left(\phi^{(s+1)}\right)_{i}^{j+1}-\left(\phi^{(s)}\right)_{i}^{j+1}\right| \leq \Lambda$,

where $\Lambda$ is a small parameter.

When condition (30) is satisfied then $\left(\phi^{(s+1)}\right)_{i}^{j+1}=\phi_{i}^{j+1}$. As an initial approach we can use $\left(\phi^{(s=0)}\right)_{i}^{j+1}=\phi_{i}^{j}$.

Eq. (29) leads to the system of linear algebraic equations

$$
A_{i}^{(s)}\left(\phi^{(s+1)}\right)_{i-1}^{j+1}-B_{i}^{(s)}\left(\phi^{(s+1)}\right)_{i}^{j+1}+C_{i}^{(s)}\left(\phi^{(s+1)}\right)_{i+1}^{j+1}=-F_{i}^{(s)}, i=\overline{1, N-1}
$$

where

$$
A_{i}^{(s)}=-\frac{1}{h_{i}+h_{i+1}}\left(1+\frac{\lambda_{p l}}{\tau}\right) b\left(\left(\phi^{(s)}\right)_{i+\frac{1}{2}}^{j+1}\right)+\frac{1}{h_{i}}\left(1+\frac{\lambda_{p l}}{\tau}\right) b\left(\left(\phi^{(s)}\right)_{i-\frac{1}{2}}^{j+1}\right)+\frac{h_{i}+h_{i+1}}{2 c_{1} h_{i} a\left(\phi_{i}^{j}\right)} q_{l m},
$$




$$
\begin{aligned}
& B_{i}^{(s)}=\frac{1}{h_{i}}\left(1+\frac{\lambda_{p l}}{\tau}\right) b\left(\left(\phi^{(s)}\right)_{i-\frac{1}{2}}^{j+1}\right)+\frac{h_{i}+h_{i+1}}{2 \tau c_{1} a\left(\phi_{i}^{j}\right)}+\frac{h_{i}+h_{i+1}}{2 c_{1} h_{i} a\left(\phi_{i}^{j}\right)} q_{l m} \\
& C_{i}^{(s)}=\frac{1}{h_{i}+h_{i+1}}\left(1+\frac{\lambda_{p l}}{\tau}\right) b\left(\left(\phi^{(s)}\right)_{i+\frac{1}{2}}^{j+1}\right), \\
& F_{i}^{(s)}=\frac{h_{i}+h_{i+1}}{2 \tau c_{1} a\left(\phi_{i}^{j}\right)} \phi_{i}^{j}+\frac{\lambda_{p l}}{\left(h_{i}+h_{i+1}\right) \tau} b\left(\left(\phi^{(s)}\right)_{i+\frac{1}{2}}^{j+1}\right)\left(\phi_{i-1}^{j}-\phi_{i+1}^{j}\right)-\frac{\lambda_{p l}}{h_{i} \tau} b\left(\left(\phi^{(s)}\right)_{i-\frac{1}{2}}^{j+1}\right)\left(\phi_{i-1}^{j}-\phi_{i}^{j}\right) .
\end{aligned}
$$

The Eq. (27) is used to determine the step $h_{i+1}$ and it can be written in the form

$$
\left(h_{i+1}\right)^{2}-\tau\left(q_{l m}\right)_{0}^{j+1} h_{i+1}+\tau c_{2} c\left(\phi_{i-\frac{1}{2}}^{j}\right)\left(\phi_{i}^{j+1}-\phi_{i-1}^{j+1}+\frac{\lambda_{p l}}{\tau}\left(\phi_{i}^{j+1}-\phi_{i-1}^{j+1}-\phi_{i}^{j}+\phi_{i-1}^{j}\right)\right)=0 \text { (32) }
$$

By solving this nonlinear equation for each temporal layer, we can determine $h_{i+1}$. The system of linear algebraic Eq. (31) is solved by the Tomas' algorithm

$$
\left(\phi^{s+1}\right)_{i}^{j+1}=\alpha_{i+1}\left(\phi^{s+1}\right)_{i+1}^{j+1}+\beta_{i+1}, i=0,1, \ldots
$$

where

$\alpha_{i+1}=\frac{C_{i}^{(s)}}{B_{i}^{(s)}-A_{i}^{(s)} \alpha_{i}}, \beta_{i+1}=\frac{F_{i}^{(s)}+A_{i}^{(s)} \beta_{i}}{B_{i}^{(s)}-A_{i}^{(s)} \alpha_{i}}$.

The starting values of the coefficients $\alpha_{1}$ and $\beta_{1}$ are determined from the boundary condition (33), which have the form

$$
\alpha_{1}=\frac{R_{m} \mu c^{0}\left(\phi_{0}^{j}\right)\left(1+\frac{\lambda_{p l}}{\tau}\right)}{R_{m} \mu c^{0}\left(\phi_{0}^{j}\right)\left(1+\frac{\lambda}{\tau}\right)+h_{0}}, \beta_{1}=\frac{R_{m} \mu c^{0}\left(\phi_{0}^{j}\right)\left(\phi_{0}^{j}-\phi_{1}^{j}\right)+h_{0} p_{0}}{R_{m} \mu c^{0}\left(\phi_{0}^{j}\right)\left(1+\frac{\lambda_{p l}}{\tau}\right)+h_{0}} .
$$

\section{Numerical Results}

Numerical results with using Eq. (31), Eq. (32) were obtained with the following values of the parameters: $p_{A}=10^{4} \mathrm{~Pa}, p_{0}=10^{5} \mathrm{~Pa}, R_{m}=10^{12} 1 / \mathrm{m}, \mu=10^{-3} \mathrm{~Pa} \cdot \mathrm{s}, k^{0}=10^{-13} \mathrm{~m}^{2}, \varepsilon_{s}^{0}=0.20$, $\varepsilon_{s_{0}}=0.0076, \beta=0.13, \delta=0.57$. These values are typical for cake filtration process $[5,6]$.

Some results are graphically shown below. The dynamics of the compression pressure and the pressure in the liquid phase at a given point $x$ for different values of the relaxation time $\lambda_{p l}$ is shown in Figure 2(a). At constant values of the all other parameters increasing in the relaxation time leads to a slow grows of the compression pressure, i.e. in the dynamics of the pressure one can see the lag. This phenomenon effects on all other filtration characteristics.

On the contrary, in pressure for the liquid phase relaxation effects lead to the advanced dynamics (Figure 2(b)). Within the framework of assumptions made above we have $p_{0}=p_{l}+p_{s}$. Comparing two figures, Figure 2(a) and Figure 2(b), we can check the implementation of the $p_{0}=p_{l}+p_{s}$. By summing values $p_{l}$ and $p_{s}$ from corresponding curves of Figure 2(a) and Figure 2(b) we obtain $p_{0}$. In addition, the fulfilment of the condition $p_{0}=p_{l}+p_{s}$ shows us correctness of numerical computations. 

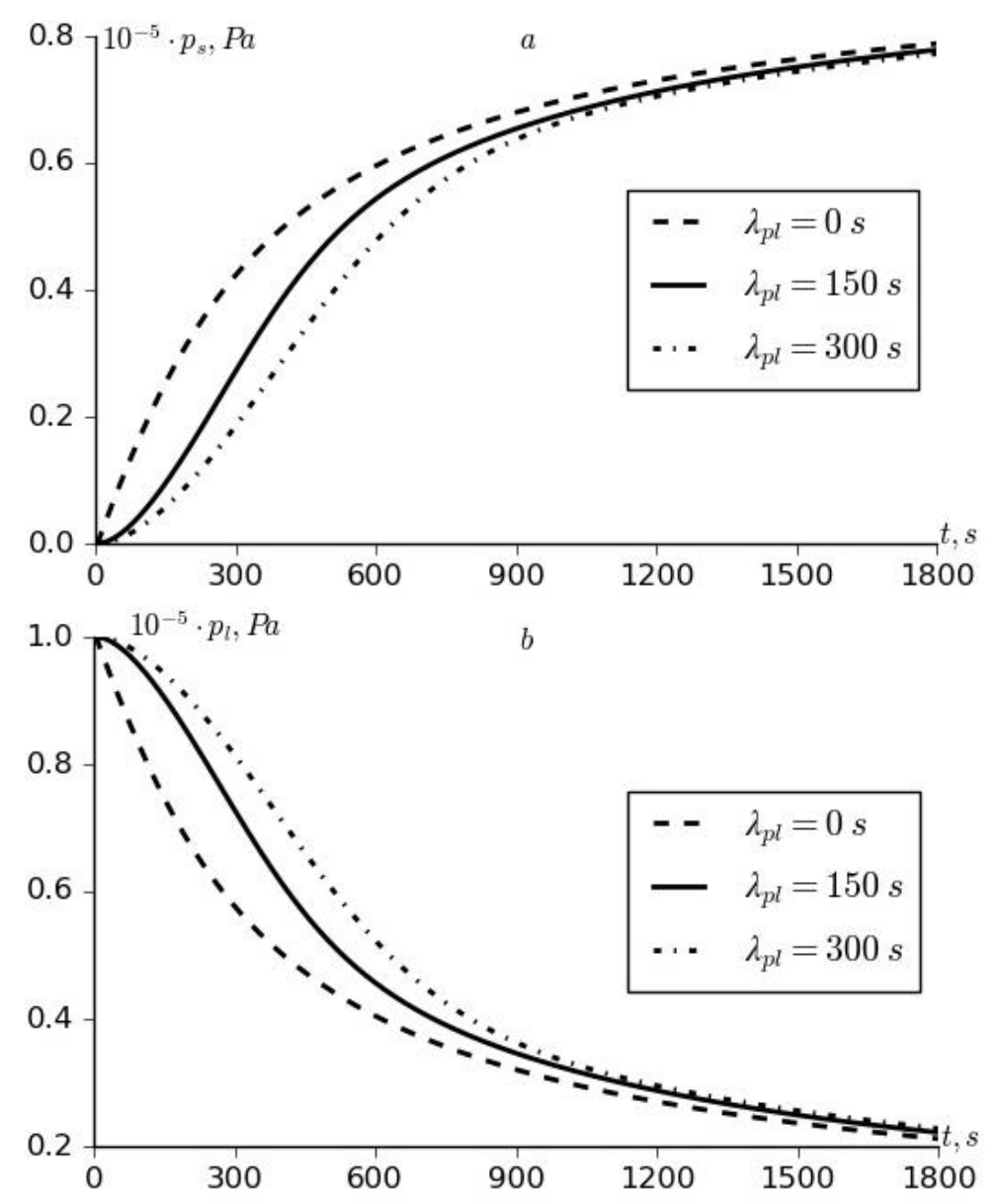

Fig. 2. Dynamics of $p_{s}(a), p_{l}(b)$ in $x=0.02 \mathrm{~m}$

Dynamics of $\varepsilon_{s}$ and $k / k_{0}$ at a given point of the cake is also determined (Figure 3 ). The curves indicate that the relaxation phenomena lead to slow dynamics of $\varepsilon_{s}$ and faster dynamics of $k / k_{0}$. In other words, relaxation phenomena in filtration law cause slow dynamics of the volume fraction of particles, i.e. solidosity. As a result, relative permeability of the cake is greater at a given point and given time. So, one can conclude that relaxation phenomena increase fluid relative velocity through cake for a given pressure $p_{0}$ and, consequently, for a given pressure gradient.

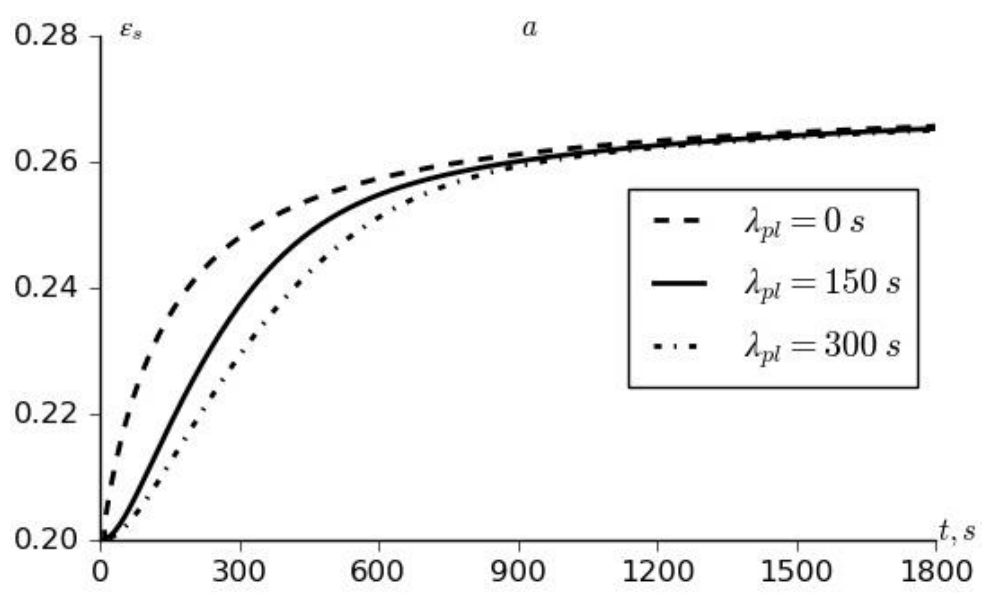




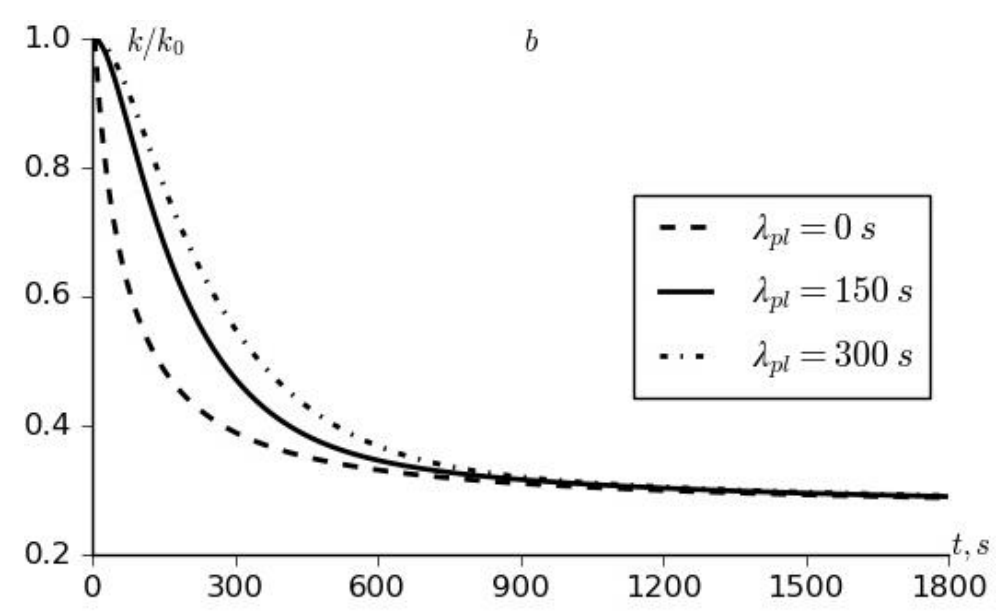

Fig. 3. Dynamics of $\varepsilon_{s}(a), k / k_{0}(b)$ in $x=0.02 \mathrm{~m}$.

The growth of the cake thickness for different values of the relaxation time $\lambda_{p l}$ is shown in Figure 4. As one can see the increasing of relaxation time $\lambda_{p l}$ leads to the faster growth of the cake thickness at all other constant conditions. In other words, relaxation effects cause more intensive transfer of particles from suspension to the cake. It, in turn, alters all other filtration characteristics, such as fluid pressure distribution, compressive pressure, porosity and consolidation of the cake, fluid flow rate through the cake and effective hydrodynamic resistance.

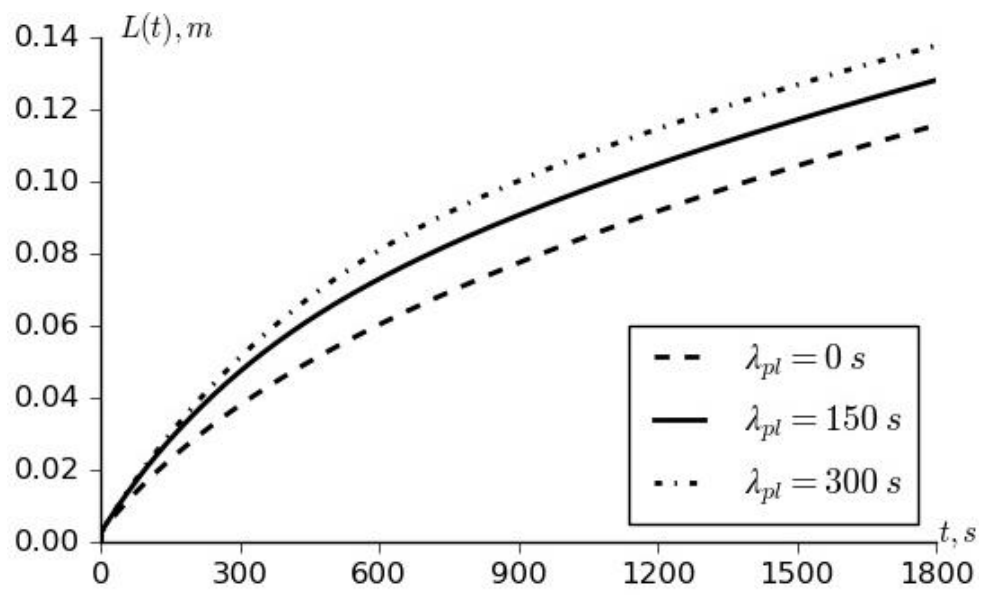

Fig. 4. Dynamics of the cake thickness

Dynamics of $q_{l m}$ at different relaxation time $\lambda_{p l}$ is shown in Figure 5(a). As we see from Figure 5(a) with relaxation phenomena $q_{l m}$ has more intensive dynamics for a same time period, duration of which is characterized by the value of $\lambda_{p l}$. Outside of this time period values of $q_{l m}$ for different $\lambda_{p l}$ are close, that indicates the ending of the influence of $\lambda_{p l}$. So, transient relaxation period is completed.

With using curves of Figure 5(a) total filtrate flow rate $Q_{l m}$ is determined as

$Q_{l m}=\int_{0}^{t} q_{l m} d t$

Some representative curves of $Q_{l m}$ are shown in Figure 5(b). They show that for greater relaxation times $\lambda_{p l}$, the total mass of filtered liquid for a given time is greater. 
All analyzed results show that relaxation phenomena cause considerable change of filtration characteristics.

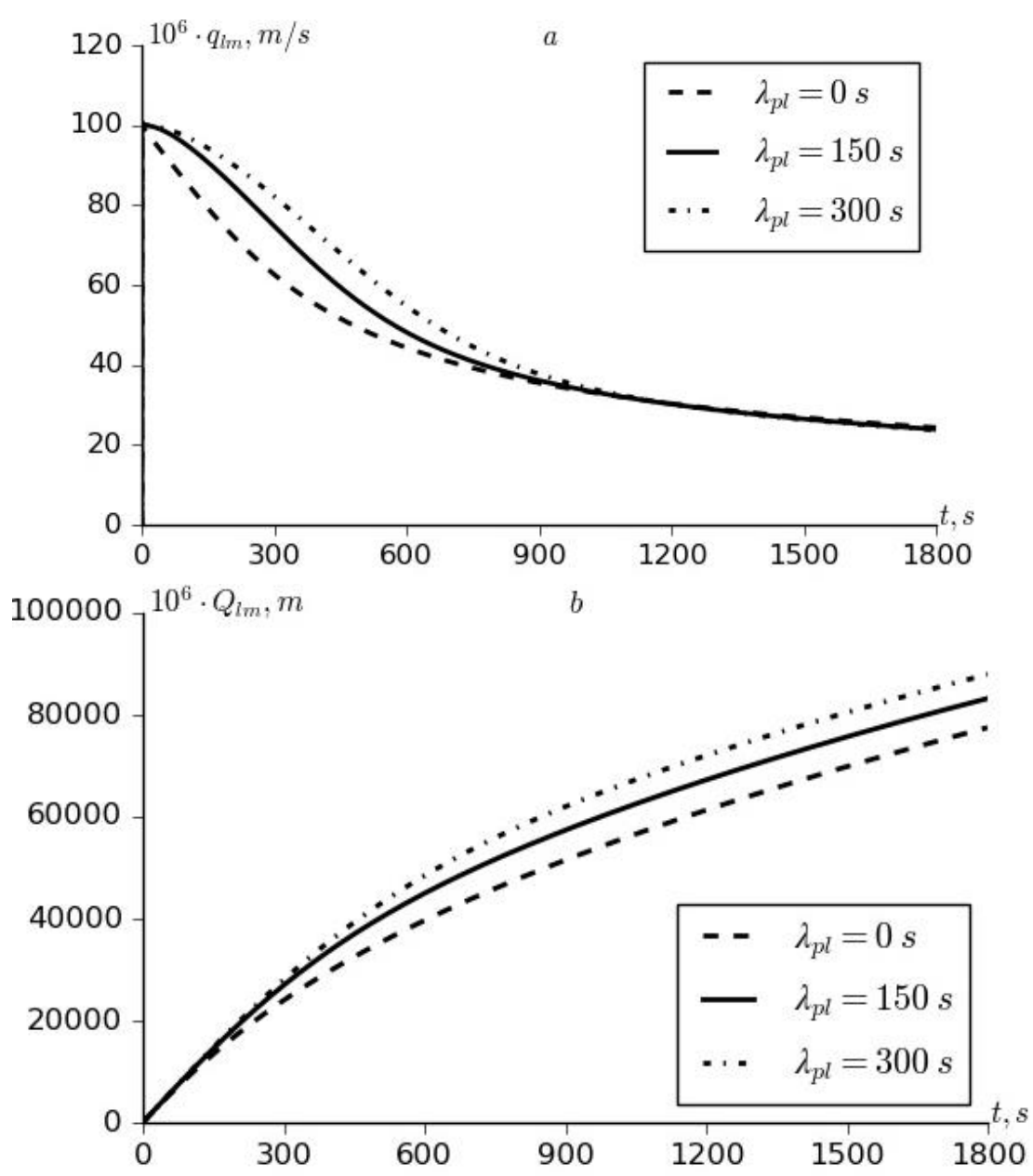

Fig. 5. Dynamics of $q_{l m}(a), Q_{l m}(b)$

\section{Conclusions}

In the paper, relaxation cake filtration equations are derived. Filtration law for liquid/particle relative velocity is written in differential relaxation form with triple relaxations, one - with respect to superficial liquid velocity $q_{l}$, the second one-with respect to the superficial particle velocity $q_{s}$, the third one-with respect to liquid pressure gradient $\partial p_{l} / \partial x$. There the only last case was analysed. Using power law dependences of solidosity $\varepsilon_{S}$ and permeability $k$ of the cake with respect to compressive pressure $p_{s}$, as well as relations between $p_{l}$ and $p_{s}$ a relaxation filtration equation is derived, that is written with respect to $p_{s}$. Using balance law on the common boundary of suspension and cake, a relaxation equation for cake thickness $L(t)$ is also derived.

Equations are numerically solved. Influence of the relaxation parameter $\lambda_{p l}$ on filtration characteristics is established. It is shown that relaxation effects lead to the slow dynamics of $p_{s}$ and more intensive dynamics of $p_{l}$ at a given point. Similarly, relaxation phenomena lead to slow dynamics of $\varepsilon_{s}$ and faster dynamics of $k / k_{0}$. So, relaxation phenomena in filtration law cause slow dynamics of the solidosity and faster dynamics of relative permeability. All of obtained results indicate that relaxation phenomena in filtration law increase fluid relative velocity through the cake for a given inlet pressure $p_{0}$ and, as a consequence, for a given pressure gradient. 
It was established that the increasing of the relaxation time leads to the faster growth of the cake thickness, i.e. relaxation phenomena cause more intensive sedimentation of the particles from suspension to the cake. Relaxation phenomena alter also other filtration characteristics such as fluid pressure distribution, compressive pressure, porosity and consolidation of the cake, fluid flow rate through the cake effective hydrodynamic resistance etc.

Dynamics of $q_{l m}$ and total filtrate flow rate $Q_{l m}$ also analysed for different relaxation times. It was shown that with increasing of relaxation phenomena dynamics of $q_{l m}$ becomes more intensive. This dependence we can observe in dynamics of $Q_{l m}$ also.

On the basis of obtained results one can conclude that relaxation phenomena cause considerable change of filtration characteristics.

\section{Acknowledgements}

This work is supported by Ministry of innovative development of the Republic of Uzbekistan, research grant OT-F4-64. Authors greatly appreciated two anonymous reviewers for their extremely detailed and useful comments.

\section{References}

[1] Ruth, B. F. "Studies in Filtration III. Derivation of General Filtration Equations." Industrial \& Engineering Chemistry 27, no. 6 (1935): 708-723.

https://doi.org/10.1021/ie50306a024

[2] Parn-Anurak, Supalak, and Thomas W. Engler. "Modeling of Fluid Filtration and near-Wellbore Damage along a Horizontal Well." Journal of Petroleum Science and Engineering 46, no. 3 (2005): 149-160. https://doi.org/10.1016/j.petrol.2004.12.003

[3] Windarto, AgusYodiGunawan, Pudjo Sukarno, and Edy Soewono."Modeling of Mud Filtrate Invasion and Damage Zone Formation." Journal of Petroleum Science and Engineering 77, no. 3-4 (2011): 359-364.

https://doi.org/10.1016/j.petrol.2011.04.011

[4] Ytrehus, Jan David, Pierre Cerasi, and Nils Opedal. "Dynamic Fluid Erosion on Filter Cakes." In 10th SPE International Conference and Exhibition on European Formation Damage. 2013.

[5] Tien, Chi. Principles of Filtration. Elsevier, 2012.

[6] Tien, Chi. Introduction to Cake Filtration. Elsevier Science, 2006. https://doi.org/10.1016/B978-044452156-9/50010-2

[7] Olivier, J., J. Vaxelaire, and E. Vorobiev. "Modelling of Cake Filtration: An Overview." Separation Science and Technology 42, no. 8 (2007): 1667-1700. https://doi.org/10.1080/01496390701242186

[8] Vyalov, S. Rheological Fundamentals of Soil Mechanics, 1st Edition. Elsevier Science, 1986. https://doi.org/10.1016/B978-0-444-42223-1.50005-3

[9] Fedotkin, I. M., Vorob'ev, E. I., and V'yun, V. I. Hydrodynamic Theory of Filtration of Suspensions. VishchaShkola, 1986.

[10] Fedotkin, I. M. Separation of suspensions and hyperfiltration: Theory, calculation, design. Technique, 1972.

[11] Mazlan, Nurul Ain, Khairul Faezah Md Yunos, MohdNazliMohdNaim, and AzhariSamsuBaharuddin. "Performances of Sandwich Membrane in Reclamation of Water from Final Discharged POME." Journal of Advanced Research in Materials Science 47, no. 1 (2018): 1-8.

[12] MohdSyafiqSharip, NorazlianieSazali, Ahmad Shahir Jamaludin, Muhammad Atif Mohamed Azmi, Farhana Aziz, and Wan Norharyati Wan Salleh. "Current Advancement by Membrane Technology: A Review." Journal of Advanced Research in Fluid Mechanics and Thermal Sciences 59, no. 2 (2019): 283-290.

[13] Corapcioglu, M. Yazuv, and Nelly M. Abboud. "Cake Filtration with Particle Penetration at the Cake Surface." SPE Reservoir Engineering 5, no. 3 (1990): 317-326.

https://doi.org/10.2118/19021-PA

[14] Atsumi, Kunio, and Tetsuo Akiyama. "A Study of Cake Filtration." Journal of Chemical Engineering of Japan 8, no. 6 (1975): 487-492. https://doi.org/10.1252/icej.8.487

[15] Shirato, M., and A. Tsutomu. "Verification of Internal Flow Mechanism Theory of Cake Filtration." Filtration and Separation 9 (1972): 290-297. 
[16] Smiles, De, and Rosenthal Mj. "The Movement of Water in Swelling Materials." Soil Research 6, no. 2 (1968): 237. https://doi.org/10.1071/SR9680237

[17] Smiles, De, and Hg Poulos. "The One-Dimensional Consolidation of Columns of Soil of Finite Length." Soil Research 7, no. 3 (1969): 285-291. https://doi.org/10.1071/SR9690285

[18] Chen, Yi-Shun, and Shu-San Hsiau. "Cake Formation and Growth in Cake Filtration." Powder Technology 192, no. 2 (2009): 217-224. https://doi.org/10.1016/i.powtec.2008.12.014

[19] Vorobiev, E. "Derivation of Filtration Equations Incorporating the Effect of Pressure Redistribution on the CakeMedium Interface: A Constant Pressure Filtration." Chemical Engineering Science 61, no. 11 (2006): 3686-3697. https://doi.org/10.1016/i.ces.2006.01.010

[20] Silva, Kelly Cristina Da, Isadora De Jesus Da Silva, Luís AméricoCalçada, and Cláudia Miriam Scheid. "The Effect of Previous Sedimentation on the Filtration and Mud cake Properties of Newtonian and Non-Newtonian Fluids." Powder Technology 346 (2019): 9-16. https://doi.org/10.1016/i.powtec.2019.01.038

[21] Smiles, De. "Steady Flow Experiments in Saturated Clays." Soil Research 7, no. 2 (1969): 91. https://doi.org/10.1071/SR9690091

[22] Smiles, De. "A Theory of Constant Pressure Filtration." Chemical Engineering Science 25, no. 6 (1970): $985-996$. https://doi.org/10.1016/0009-2509(70)85043-6

[23] Wakeman, R. G. "A numerical integration of the differential equations describing the formation of and flaw in compressible filter cakes." Trans. Inst. Chem. Eng. 56, no. 4 (1978): 258-265.

[24] Iritani, Eiji, Nobuyuki Katagiri, and Haruki Masuda. "Simplified Estimate of Cake Porosity in Dead-End Ultrafiltration of Protein Solution." Journal of Chemical Engineering of Japan 51, no. 7 (2018): 589-595. https://doi.org/10.1252/jcej.18we033

[25] Buevich, Yu. A., V. A. Ustinov, and B. Khuzhayorov. "Nonsteady Transfer in Disperse and Heterogeneous Media." Journal of Engineering Physics 56, no. 5 (1989): 552-558. https://doi.org/10.1007/BF01297605

[26] Khuzhayorov, B. Kh. "Modeling of relaxation phenomena in the motion of non-Newtonian fluids." Uzbek Journal Problems of Mechanics 3-4 (1994): 55-56.

[27] Zubarev, A. Yu., and B. Khuzhayorov. "Relaxational Filtration." Journal of Engineering Physics 55, no. 3 (1988): 10201024. https://doi.org/10.1007/BF00870487

[28] Khuzhayorov, B. Kh., and Makhmudov, Zh. M. Mathematical models of the filtration of non - homogeneous liquids in porous media. Tashkent, Fan, 2014.

[29] Iwata, Masashi, and Toshiro Murase. "Expansion and Stress Relaxation of Expressed Cake." Drying Technology 11 , no. 4 (1993): 749-767. https://doi.org/10.1080/07373939308916862

[30] Loginov, Maksym, FlorianeDoudiès, Nicolas Hengl, Frédéric Pignon, and Geneviève Gésan-Guiziou. "Influence of Membrane Resistance on Swelling and Removal of Colloidal Filter Cake after Filtration Pressure Release." Journal of Membrane Science 595 (2020): 117498. https://doi.org/10.1016/i.memsci.2019.117498

[31] Khuzhayorov, B. Kh. Filtration of non - homogeneous liquids in porous media. Tashkent, Fan, 2012.

[32] Barenblatt, G. I., Yu. G. Mamedov, A. Kh. Mirzadzhanzade, and I. A. Shvetsov. "Nonequilibrium Effects in the Filtration of Viscoelastic Liquids." Fluid Dynamics 8, no. 5 (1975): 742-748. https://doi.org/10.1007/BF01023573

[33] Alishayev, M. G. "On nonsteady filtration with pressure relaxation." Hydromechanics 111, no. 3 (1974): 166-171.

[34] Molokovich, YuriiMatveevich. "On the theory of linear filtration with allowance for relaxation effects." IzvestiyaVysshikhUchebnykhZavedenii. Matematika 5 (1977): 66-73.

[35] Khuzhayorov, B. Kh. "Macroscopic simulation of relaxation mass transport in a porous medium." Fluid Dynamics 39, no. 5 (2004): 693-701. https://doi.org/10.1007/s10697-005-0003-x

[36] Samarskiy, A. A., and Vabishchevich, P. N. Computational Heat Transfer. Wiley, 1995.

[37] Caldwell, J., and Y. Y. Kwan. "Numerical Methods for One-Dimensional Stefan Problems." Communications in Numerical Methods in Engineering 20, no. 7 (2004): 535-545. https://doi.org/10.1002/cnm.691 